\title{
Abstracts from the 1st Croatian Congress on Ultrasound in Gynecology and Perinatology, 10-11 November 2018, Zagreb, Croatia
}

\author{
First Trimester Screening for Preeclampsia in Advanced Age \\ Primiparous Women \\ ${ }^{1}$ Vesna Elveđi-Gašparović, ${ }^{2}$ Petrana Beljan, ${ }^{3}$ Saša K Oguić, ${ }^{4}$ Snježana Škrablin \\ ${ }^{1,2,4}$ Department of Obstetrics and Gynecology, University Hospital Centre Zagreb and School of Medicine, \\ University of Zagreb, Zagreb, Croatia \\ ${ }^{3}$ Department of Clinical Biochemistry, University Hospital Centre Zagreb, Zagreb, Croatia
}

\section{INTRODUCTION}

Preeclampsia is a devastating cardiovascular disorder of pregnancy, affecting 5-10\% of all pregnancies and responsible for 60,000 maternal and over 500,000 fetal deaths worldwide each year. The lack of understanding the complex interplay among immune, vascular, renal and angiogenic mechanism and subsequent systemic endothelial and vascular dysfunction makes early diagnosis and prevention of preeclampsia almost impossible. As delivery remains the only choice of treatment, the early prediction will offer timely therapeutic intervention, so extensive research efforts have been done in the identification of biomarkers which will facilitate early prediction of the disease.

\section{PURPOSE}

We investigate the potential value of maternal serum copeptin and placental protein 13 (PP13) levels at 11-13 weeks' gestation in the prediction of preeclampsia in a case-control study.

\section{MATERIALS AND METHODS}

The study included 150 primiparous pregnant women $\geq 35$ years who received antenatal obstetric care in our hospital. Maternal serum concentration of copeptin and PP13 were measured at 11-13 weeks' gestation in cases of PE $(\mathrm{n}=11)$ and controls $(\mathrm{n}=139)$. Comparison of biomarker levels between the groups was done using the Mann-Whitney test. The level of significance was $p<0.05$. We used the ROC curves to find a cut-off value for the biomarkers.

\section{RESULTS}

The mean copeptin plasma levels were $0.32 \pm 0.25 \mathrm{ng} / \mathrm{mL}$ in control and $0.47 \pm 0.18 \mathrm{ng} / \mathrm{mL}$ in the PE group, respectively. Women who subsequently developed PE showed serum concentration of copeptin significantly higher compared to controls $(p=0.012)$. PP13 plasma level was found decreased in the PE group $(93.4 \pm 6.91 \mathrm{pg} / \mathrm{mL}$ ) compared to controls (118.5 \pm 13.02$)$ In PE group newborns have significantly lower birth weight $(p=0.031) .64 \%$ PE pregnancies were complicated with IUGR $(p<0.001)$. Logistic regression model for PE using primiparity, maternal age and biomarkers confirmed a significant contribution for the combination of two biomarkers for prediction of PE $(p=0.03)$

\section{CONCLUSION}

Maternal serum copeptin and PP13 levels in the first trimester is by far a promising method for early detection of PE in advanced age nulliparous women. 
Impaired placental histoarchitectonics, inappropriate nidation, and consequential malplacentation occur due to biotopic (endometrium-decidua) and autonomic cause (blastocyst) and their interaction that results in abnormal biohumoral and morphological response that yields a pathologic ultrasound pattern. Bleeding in early pregnancy is an early clinical sign of inappropriate placentation, providing important information for subsequent development of late complications of pregnancy, in particular, preeclampsia and morbid invasive malplacentation as serious obstetric problems with a rising incidence. Malplacentation is associated with frequent intrauterine procedures, uterine inflammation, a high number of cesarean sections and myoma enucleation. Hypoxic-ischemic states in pregnancy requiring pharmacotherapy induce biochemical-morphological modulations in fetal (perinatal) programming and are associated with short- and long-term sequels in the child.

Damaged endometrium and myometrium along with the absence of Nitabuch fibrinoid effect make the normal placentation processes impossible. Abnormal placentation is indicated by an angiogenic imbalance between elevated values of the soluble form-like tyrosine kinase (sFlt1) biomarker and decreased values of the placental growth factor (PlGF), a vascular endothelial growth factor analog, as the most relevant markers predicting preeclampsia. In addition, the function of the placental protein 13, pregnancy-associated plasma protein-A, myostatin, irisin, leptin, afamin, and placental vitamin D is modulated; thus these are also first-trimester predictors of malplacentation, preeclampsia events, intrauterine growth restriction, and poor perinatal outcome. In preeclampsia, abnormal remodeling of spiral arteries occurs, i.e., conversion of early trophoblast invasion and failure of physiological second wave of trophoblast invasion into uterine spiral arteries by the 18th week of gestation, cytotrophoblast invasion with conversion from epithelial to endothelial phenotype, so-called pseudovasculogenesis (antiangiogenesis) with development of acute atheromatosis of placental vasculature.

Ultrasound morphological signs range from innocent to retrochorionic ablation and hematoma, later with Doppler notching sign and significantly high resistance in uterine arteries ('arterialization'). Invasive malplacentation is characterized by the absence of normal placentation contours, the absence of myometrium contours with dilated blood vessels and numerous vascular lakes, and low implantation of the gestational sac.

Current biochemical tests (sFlt1)/PlGF) has $>90 \%$ predictive value in early pregnancy, whereas in the second trimester the biomarker quotient correlates directly with disease severity, complications, and short-and long-term outcome for both the mother and the child/children. Doppler and other ultrasound variables (RI AUT and notching) are monitored in the second trimester. In case of invasive, and especially prevail malplacentation, diagnosis must be made antenatally to enable timely planning of the operative procedure. Doppler ultrasound and morphometric follow-up of impaired pregnancy, from early placentation to biophysical profile in late pregnancy is the only reliable method in these cases.

\title{
Ultrasound Elastography in Obstetrics and Gynecology
}

Maryna Kharchenko

\author{
Private Clinic Veritas, Zagreb, Croatia
}

Ultrasound elastography (USE) is a noninvasive ultrasound method for analysis and visualization of soft tissue stiffness and elasticity, so-called virtual or visual palpation. Elasticity is the mechanical property of soft tissues that depend on micro- and macro-structure and is unrelated to tissue echogenicity. Therefore, ultrasound elastography is a method for early detection and monitoring of tumors and diffuse pathological changes in various organs as it provides additional information on tissue structure and behavior under compression. Elasticity imaging methods were introduced into clinical practice around 10 years ago and are being actively developed, both in terms of technology and areas of clinical application. The European Federation of Societies for Ultrasound in Medicine and Biology (EFSUMB) and the World Federation for Ultrasound in Medicine and Biology (WFUMB) have already provided evidence-based guidelines for clinical application of ultrasound elastography in the differential diagnosis of benign and malignant breast and thyroid tumors as well as diffuse liver diseases. Numerous studies on the use of ultrasound elastography in gynecology and obstetrics expand existing knowledge on physiological and pathological processes and provide additional capability for early diagnosis. Promising results are obtained by elastographic cervical examination studies with regard to prediction of premature birth and successful induction of labor. Placental elasticity becomes a new and additional ultrasound marker for assessing placental function in pathological pregnancies. The application of ultrasound elastography in the differential diagnosis of uterine fibroids, adenomyosis, endometrial pathology and in the field of gynecological oncology is actively investigated as well. The goal of this presentation is therefore to show principles of various ultrasound elastography methods, their benefits, and limitations for clinical application in gynecology and obstetrics. 
Sonoembryology

Asim Kurjak

Director of Ian Donald School; President of International Academy of Perinatal Medicine

\title{
OBJECTIVE
}

To evaluate the role of 3D, 4D and color Doppler ultrasound in the assessment of early human development.

\section{MATERIALS AND METHODS}

Three hundred eighty women with uncomplicated early pregnancy between 5 weeks and 14 weeks were evaluated by 3D, 4D, and color Doppler ultrasound.

\section{RESULTS}

Regression analysis revealed the exponential rise of the gestational sac volume with gestational age throughout the first trimester. An exponential rise of the yolk sac volume was noticed between gestational weeks 5 and 8, followed by a gradual increase of the yolk sac volume between 8 weeks and 10 weeks. After reaching the plateau from 10 to 11 weeks, yolk sac volume started to decrease. Three-dimensional ultrasound was used to study nuchal translucency in 120 patients between 10 weeks and 14 weeks of gestation. Multiplanar imaging allowed an appropriate mid-sagittal section of the fetus and clear distinction of the nuchal region from the amniotic membrane in all the examined patients. This enabled us to obtain nuchal translucency measurements in $100 \%$ of cases. Rotation of the embryo and close scrutiny of the volume allowed systematic review of anatomic structures such as cord insertion, limb buds, cerebral cavities, stomach, and bladder.

\section{CONCLUSION}

Three-dimensional ultrasound is advantageous for studying normal embryonic and/or fetal development, as well as providing information for families at risk for specific congenital anomalies by confirming normality. Three-dimensional ultrasound imaging complements pathologic and histological evaluation of the developing embryo rising a new term: 3D sonoembryology. It is expected that interesting data on fetal behavior will be collected with the introduction of 4D sonography.

\section{Can Ultrasound Identify Motor Changes which are Antecedent to Cerebral Palsy?}

\author{
Asim Kurjak \\ Director of lan Donald School; President of International Academy of Perinatal Medicine
}

Fetal behavior refers to the fetal activities observed or recorded with ultrasonographic equipment. Behavior can be spontaneous, generated by the fetus itself, or elicited in response to an external stimulus such as vibroacoustic stimulation. Analysis of the fetal dynamics in comparison with morphological studies has led to the conclusion that fetal behavioral patterns directly reflect developmental and maturational processes of the fetal central nervous system (CNS). In addition, there is a carryover effect of movements from prenatal to postnatal life. There were no movements observed in fetal life that were not present in neonatal life.

Furthermore, prenatal-neonatal continuity exists even in subtle, fine movements such as facial mimics. Altered quality of fetal movements might reveal the structural or functional impairment of the fetal central nervous system. Improvement of four-dimensional (4D) technology enabled the introduction of Kurjak Antenatal Neurological Test (KANET), scoring test for the assessment of the fetal behavior. Our preliminary results have confirmed the usefulness of this test in fetal behavior assessment. The KANET test has the potential to detect and discriminate normal from borderline and abnormal fetal behavior in normal and in high-risk pregnancies, which means that it could become a valuable diagnostic tool for fetal neurological assessment. Over 100 fetuses from pregnancies with threatened preterm labor have been studied using KANET in our multicentric program. Recently study with the largest number of fetuses (620 fetuses) where prenatal KANET test has been applied was published. Among the fetuses with abnormal KANET score, most frequently presented were fetuses from the threatened preterm delivery group. Preterm labor accounts for $75 \%$ of perinatal mortality and over $50 \%$ of perinatal morbidity. Although preterm survives, they are at increased risk of neurodevelopmental impairment. It has been shown that fetal behavior differs in preterm than term infants. Further, fetal behavior differs in neurologically compromised compared to normal fetuses. There are developmental differences between fetuses threatening to deliver early and other high-risk fetuses. Preterm infants are at higher risk for cerebral palsy due to prematurity, intrauterine growth restriction, infection or multiple pregnancies. Pediatricians know that they need to wait until the age of 6 months postnatally to diagnose a severe $\mathrm{CP}$ and at least 24 months or even longer for a minor non-disabling CP. KANET test and behavioral assessment give new hope in the attempt of early diagnosis of cerebral palsy and provide identification of fetuses at neurological risk from pregnancies with threatened preterm labor. 
Abstracts

\section{Fetal Arrhythmias}

Rajka Lulić Jurjević

Dječja bolnica Srebrnjak, Medicinski fakultet Osijek

Irregularities of fetal cardiac rhythm are detected in approximately $1-2 \%$ of pregnancies. In most cases, these are atrial extrasystoles, which have little clinical relevance.

However, some types of cardiac dysrhythmias are of clinical significance because they can cause fetal compromise, which may lead to the death of the affected fetus. Both, sustained fetal tachycardia, atrial flutter, ventricular tachycardia, and atrioventricular heart block are associated with fetal and postnatal morbidity and mortality.

Supraventricular tachycardia (SVT) usually starts and stops suddenly with a monotonous heart rate of 180-280/minute. Sustained SVT can result in hydrops or death and represents a fatal medical emergency.

Atrial flutter is unusual fetal tachycardia. The atrial rate is 300 to $500 \mathrm{bpm}$ with varying ventricular response. Ventricular rate shows significant variability from 170 to $400 \mathrm{bpm}$.

Echocardiography enables evaluation of hemodynamic consequences of tachycardia and detection of associated structural heart abnormalities. Correct assessment of tachycardia type and its complications are crucial for the appropriate management of this condition. Fetuses presenting before 36 weeks of gestation can successfully be treated by transplacental administration of antiarrhythmic medications. In contrast, mature fetuses can be delivered and treated after birth.

Transient bradycardia is in response to pressure from the transducer and a normal fetal heart rate response and should not be confused with the pathologic bradycardia that results in sustained slow heart rates.

The pathologic bradycardia group is made up of second and third-degree atrioventricular block. Third degree or complete heart block is present when there is a dissociation between the atrial and ventricular complexes and the atrial rate is faster than the ventricular rate.

Approximately $50 \%$ of fetuses with complete heart block have significant structural heart disease, specifically atrioventricular septal defects, corrected transposition of the great arteries, cardiac tumors or cardiomyopathy. In fetuses with complete heart block without structural defects, there is a high incidence of maternal connective tissue disease.

Treatment of fetal dysrhythmias is a complex process and involves a multidisciplinary approach.

\section{Fetal Echocardiography from the Angle of Gynecologist}

Ulla Marton

Polyclinic, Dr Marton, A Hebranga, Zagreb, Croatia

During the last three decades, imaging of the fetal heart has evolved into a sophisticated and widely practiced clinical tool, but most heart diseases still go prenatally undetected despite routine fetal ultrasound evaluations. Over the last decade, tremendous advances in fetal cardiac imaging, including three-dimensional imaging, promise to revolutionize both the prenatal detection and diagnosis of congenital heart diseases. Image resolution continues to improve year after year, allowing earlier and better visualization of fetal cardiac structures. Introduction of three-dimensional imaging of the fetal heart may improve the detection of outflow tract abnormalities and facilitate comprehension of complex forms of congenital heart diseases (CHD). Improvement of prenatal noninvasive diagnosis by the end of the first trimester leads to a better detection rate of fetal congenital heart disease. In this paper, highlight in on the potential of early fetal assessment by the end of first and begging of the second trimester. On the other hand, other imaging modalities, which had been introduced in clinical practices, such as Doppler tissue imaging and magnetic resonance imaging of the fetus, continue to evolve and to complement two- and three-dimensional sonographic imaging of the fetal heart. As a result of these ongoing progress in prenatal detection and technical improvements, assessment of $\mathrm{CHD}$, has exciting potential. ${ }^{1}$

\section{IMPACT OF CONGENITAL HEART DISEASES: EPIDEMIOLOGY, POPULATION AT RISK}

Prenatal detection of fetal congenital heart defects still remains the most problematic prenatal diagnostic issue. ${ }^{2} \mathrm{Major} \mathrm{CHD}$ is the most common severe congenital malformations, with an incidence of about 7-8 in a thousand live births, whenever complete ascertainment is done and minor lesions are excluded. ${ }^{2,3}$ Congenital heart anomalies have a significant effect on affected children's life and the life of their families with up to $25-35 \%$ mortality rate during pregnancy and the postnatal period, and it is during the first year of life when the $60 \%$ of this mortality occurs. Major CHD is responsible for nearly $50 \%$ of all neonatal and infant deaths due to congenital anomalies, and it is likely to be significantly higher if spontaneous abortions are considered. Although it seems to be that CHD uses to appear isolated, they are frequently associated with other defects, chromosomal anomalies, and genetic syndromes and extracardiac anomalies. Their incidence is 6 times greater than chromosomal abnormalities and 4 times greater than neural tube defects. ${ }^{2-4}$ Unlike both, chromosomal and neural tube defects, for which there has been established an extensive prenatal screening program, congenital heart diseases are often not identified until the infant is born. The importance of early prenatal diagnosis of congenital heart disease is further emphasized by the fact that 20-35\% of perinatal deaths are due to congenital heart disease..$^{2-5}$ Nonetheless, congenital cardiac defects are still among the most 
frequently missed abnormalities by prenatal diagnostic structural evaluatuion. ${ }^{5}$ Although the at-risk population-based approach has been introduced and it showed to be crucial in decreasing disease, prenatal diagnosis of CHD remains largely a scenario of too much effort for too few diagnoses. Medical professionals involved in prenatal detection program need to re-examine the reasons for this shortfall and re-define new strategies to improve the efficacy of all effort. It should be kept in mind that most of all congenital heart defects (90\%) occur in low-risk population/mothers. The way forward to increase prenatal detection of CHD is to improve the effectiveness of routine screening programs so that a higher number of cases from low-risk populations are referred for a specialized scan. The positive aspect of screening for cardiac defects compared with other anomalies (trisomy's-Down syndrome, neural tube defects) is that it does not automatically result in termination of pregnancy in most cases. It should be always kept in mind that it is one of the structural anomalies where optimizing management of the fetus / neonate in the perinatal period could improve drastically the outcome. Improvement in the rate of morbidity/mortality has been clearly shown as a result of prenatal diagnosis in the outcome of all forms of CHD which are dependent on the patency of the arterial duct in the immediate postnatal period, being the transposition of the great arteries the single most important lesion to diagnose prenatally. ${ }^{6}$ All these reasons emphasize the role of ultrasonography in the prenatal diagnosis of congenital heart abnormalities, and most major CHD can be prenatally diagnosed by detailed transabdominal second-trimester echocardiography at 20-24 weeks' gestation..$^{2,4,7-9}$ The identification of pregnancies at high-risk for CHD needing a referral to specialist centers is of paramount importance to reduce the rate of overlooked defects. ${ }^{9,10}$ However, the main problem in prenatal diagnosis of CHD is that the majority of cases take place in pregnancies with no identifiable risk factors. Therefore, there is wide agreement that cardiac ultrasound screening should be introduced as an integral part of the routine scan at 20-22 week. Although recent technology advance with improved resolution has allowed fetal heart evaluation from 17th to 18th weeks of gestation or even earlier with transvaginal ultrasonography, the prenatal diagnosis of congenital heart defects is still a challenge for the modern obstetric ultrasonographer. However, first-trimester and early second trimester (13th and 14th weeks of gestation) diagnosis are increasingly becoming possible. ${ }^{7-11}$ In the 1990s, the American Institute of Ultrasound in Medicine and the American College of Radiology incorporated the four-chamber view into their formal guidelines for the screening of fetal ultrasound. ${ }^{11,12}$ We have to admit, that the accuracy of fetal echocardiography is not as precise as in newborns, and many of defects; such as small ventricular septal defects and atrial septal defects $(<6 \mathrm{~mm})$, coarctation of aorta, pulmonary stenosis and anomalous venous connections, may escape detection due to the resolution, or active and/or poorly positioned fetus.

Although early investigators found the four-chamber view to have a high sensitivity for the prenatal detection of CHD, subsequent studies have found this view to be far less sensitive. Even in the best hands, this plane may fail to detect a significant percentage of major frequently ductal-dependent CHD. Many investigators have demonstrated an incremental value of adding outflow tracts to the routine screening fetal ultrasound. ${ }^{13,14}$ When applied to the low-risk population, scrutiny of the four-chamber view allows only the detection of $40 \%$ of the anomalies while additional visualization of the outflow tracts and the great arteries increase the rate up to $60-70 \% ., 7,8$ The systematic incorporation of the four-chamber view and outflow tracts into the routine screening fetal ultrasound represents an important advance in the fetal cardiac image.

\section{STANDARD FETAL CARDIAC EXAMINATION}

The basic fetal cardiac screening examination entails an analysis of the four-chamber view, obtained from an axial plane across the fetal thorax. If technically feasible, optional views of the outflow tracts can be obtained as part of an extended cardiac screening examination. The systematic incorporation of the four-chamber view and outflow tracts into the routine screening fetal ultrasound represents an important advance in the fetal cardiac image. Summarizing the data from screening studies, a detection rate of less than $10 \%$ can be expected if the heart is not explicitly examined, a rate of $10-40 \%$ detection if the four-chamber view is visualized and a rate of $40-80 \%$ if the visualization of the great vessels is added. ${ }^{4,7,8}$

In a high-risk pregnancy, in addition to the information provided by the basic screening exam, a detailed analysis of cardiac structure and function may further characterize visceroatrial situs, systemic and pulmonary venous connections, foramen ovale mechanism, atrioventricular connections, ventriculoarterial connections, great vessels relationships and sagittal views of the aortic and ductal arches. Additional sonographic techniques can be used for this purpose, as Doppler ultrasonography or M-mode modality.

In summary, cardiovascular system malformations are clearly some of the most difficult anomalies to diagnose in prenatal ultrasonography. This is due, to the rapidity of the fetal heart rate, which hinders visualization of heart structures. A rapid heart rate superimposed by a poorly positioned fetus with fetal motion further restrict the evaluation of heart structural defects. The fetal echocardiography is very important adjunct in the management of both, "low" risk as well in patients considered to be at "high" risk pregnancies. Application of transvaginal imaging in the early second trimester allows us nowadays early and accurate detection of congenital heart disease. Early and appropriate detection of the major congenital heart diseases in utero has a considerable impact on the prenatal counseling and perinatal management.

Widespread teaching the technique will result in an increased prenatal diagnosis of congenital heart disease and should reduce the prenatal mortality and morbidity.

\section{BIBLIOGRAPHY}

1. Sklansky M. Advances in fetal cardiac imaging. Pediatr Cardiol 2004;25:307-321.

2. Office for National Statistics. Mortality Statistics. Childhood, Infancy and Perinatal. Series. DH3. Sationary Office:london.2007;40.

3. Malčić I, Dilber D. Distribucija prirođenih srčanih grešaka u Hrvatskoj i analiza ishoda liječenja. Liječ Vjesnik 2011;133:81-88 Epidemiološka studija 2002-2007.

4. Allan L, Sharland G, Milburn A, Lockhart S, Groves A, Anderson R, Cook A. Prospective diagnosis of 1006 consecutive cases of congenital heart disease in the fetus. J Am Coll Cardiol 1994;23:1452-1458. 
Abstracts

5. Garne E, Stoll C, Clementi M, and the EUROSCAN GROUP. Evaluation of prenatal diagnosis of congenital heart diseases by ultrasound: experience from 20 European registries. Ultrasound Obstet Gynecol 2001;17:386-391.

6. Bonnet D, Coltri A, Butera G, Fermont L, Le Bidois J, Kachaner J, Sidi D. Detection of transposition of the great arteries in fetuses reduces neonatal morbidity and mortality. Circulation 1999;99:916-918.

7. Allan LD. Fetal cardiology. Curr Op Obstet Gynecol 1996;8:142-147.

8. Gembruch U. Prenatal diagnosis of congenital heart disease. Prenat Diagn 1997; 17:1283-1298.

9. Perisco N, Mortalla J, Lombardi CM, et al. Fetal echocardiography at 11-13 weeks by transabdominal hight-frequency ultrasound. Ultrasound Obstet Gynecol 2011;37:296-301.

10. Levi S, Schaaps JP, De Havay P, et al. End result of routine ultrasound screening for congenital anomalies. The Belgian Multicentric study 1984-1992. Ultrasound Obstet. Gynecol 1995;5:366-371.

11. Lombardi CM, Bellotti M, Fesslova V, et al. fetal echocardiography at the time of the nuchal translucency scan. Ultrasound Obstet Gynecol 2007;29:249-257.

12. Royal College of Obstetricians and Gynaecologists. Ultrasound Screening. Royal College of Obstetricians and Gynaecologists 2000; http:/ / www.rcog.org.uk/mainpages.asp?PageID=439\#20week

13. Zidere V. First trimester fetal echocardiography. In Simpson J., Zidere V., Miller O.I. (eds.) Fetal Cardiology. Springer Cham 2018; pp 57-70.

14. Donofrio Mary T. Predicting the future: delivery room planning of congenital heart disease diagnosed by fetal echocardiography. Amer J perinatol 2018;35(06):549-552.

\title{
Is There Still a Place for Noninvasive Prenatal Diagnosis?
}

Ratko Matijevic

University of Zagreb, School of Medicine, Merkur University Hospital, Zagreb, Croatia

\begin{abstract}
There are several methods used to screen the asymptomatic low-risk population for chromosomal anomalies. The first one uses prenatal markers for trisomy phenotype, and second one is using "surrogate markers" while the third and most important one uses a marker of genotype rather than phenotype. It is well known that genetic testing of the fetus requires genetic material from the fetus, and in invasive diagnosis, we are obtaining either fetal epithelial cells in amniocentesis and mesodermal connective tissue and trophoblastic cells in chorionic villus sampling. Non-invasive fetal testing uses cell-free fetal DNA being representative of the current fetus in the current pregnancy. Methods are used for screening, and it must be pointed out that it may not identify all pregnancies with T21,18 and 13, it does not test for all chromosomal disorders and have false positive and negative results. Also, it may require additional testing and may not give an answer in some certain situations. However, it is safe, comfortable, popular and does not carry a risk for ongoing pregnancy. NIPT is a screening method and can and should be incorporated into different screening strategies. All of those will be discussed in the presentation
\end{abstract}

\section{Three-dimensional Neurosonography: Normal Development of the Cerebrum and Detection of Migration Disorders}

Eberhard Merz, Sonila Pashaj

Centre for Ultrasound and Prenatal Medicine, Frankfurt, Germany

\section{INTRODUCTION}

The prenatal development of the cerebral cortex can be divided into 3 stages: 1. cell proliferation, 2. cell migration, and 3. cortical organization.

Neural proliferation occurs between the second and the fourth month of gestation. During that time both, the neuronal and glial precursors are generated. The neurons and glial cells develop from their neuroblast precursors in the ventricular and subventricular zones. Neuronal migration takes place between the third and fifth months of gestation. During this phase, the neuronal-glial cells migrate from the proliferative zone to their final destination zone. Cortical organization starts at 22 weeks of gestation and continues until 2 years of age. Neurons differentiate into several cell types, leading to the formation of the normal cortical cytoarchitectonic pattern. ${ }^{1}$ Disorders of cortical organization result in abnormalities of gyral formation and cortical organization. ${ }^{1-3}$

\section{NORMAL ANATOMY}

Three-dimensional (3D) ultrasound with its different visualization modes allows an exact demonstration of the brain mantle, the gyri, the sulci, the ventricles, and the periventricular regions. This is particularly true when the fetus is in a vertex presentation and a high resolution transvaginal 3D ultrasound probe can be used. After a volume of the fetal brain is stored in the memory of the ultrasound unit, the volume can be rotated in all three dimensions until the fetal brain is seen in an exact upright position. 
This allows not only a precise demonstration of the median plane but also permits an exact comparison of the left and right hemisphere, including the Sylvian fissure.

\title{
PATHOLOGICAL RESULTS
}

Disorders that can be diagnosed with 3D ultrasound are abnormal neuronal/cortical formation disorders (microcephaly, megalencephaly or hemimegalencephaly), abnormal migration disorders (periventricular heterotopia, subcortical band heterotopia, classic lissencephaly, cobblestone lissencephaly) and disorders with the abnormal cortical organization (polymicrogyria, schizencephaly). The detection of an abnormal formation of the Sylvian fissure enables early diagnosis of Miller-Dieker and Walker-Warburg syndrome.

\section{CONCLUSION}

High-resolution 3D ultrasound has been shown to be as useful as MRI in diagnosing malformations of the cortical development. In comparison to MRI, ultrasound is a cheap technique that can be repeated several times to follow the dynamic development of the fetal brain. Knowledge of the normal fetal brain development and standardization of the planes of the fetal brain are necessary for the successful diagnosis of complex fetal cerebral malformations.

\section{BIBLIOGRAPHY}

1. Razek AAKA, Kandell AY, Esorogy LK et al. Disorders of cortical formation: MR imaging features. Am J Neuroradiol 2009;30:4-11.

2. Hansen PE, Ballesteros MC, Soila K, et al. MR imaging of the developing human brain. Radiographics 1993;13:21-36.

3. Barkovich J, Kuzniecky RI, Dobyns WB, et al. A classification scheme for malformation of cortical development. Neuropediatrics 1996;27:59-63.

\section{Therapeutic Interventions in Fetuses with Congenital Heart Anomaly}

Senka Mesihovic-Dinarevic

\author{
Polyclinic Eurofarm, Sarajevo, Bosnia and Herzegovina
}

Thanks to advances in imaging techniques, multidisciplinary teamwork, acquired experience and interest in fetal medicine, in the last two decade there has been an enormous step forward in the diagnosis and therapeutic interventions / TI/ in fetuses with congenital heart anomaly. Prenatal diagnosis and therapy not only requires many professional skills but entails many ethical and legislative consequences, causing a critical attitude. Therefore, the experts dealing with fetology are obliged to present their results, no matter what they are, because only in this way the contribution to the development and proper understanding of the concept could be made. Understanding the natural history of the malformation and the continual ability to refine patient selection is critical. Fetal cardiac intervention offers the potential to alter in utero anatomy and physiology so that it either is lifesaving to the fetus or results in an improved state at birth that leads to a reduction in short- or long-term morbidity or mortality. Medical and interventional treatments for select diseases and strategies for delivery room care by a well-trained multidisciplinary team with sophisticated equipment are required for improving the technical success rate and post-interventional outcomes. Therapeutic fetal cardiac interventions for congenital heart anomalies/intrauterine balloon aortic valvuloplasty, intrauterine pulmonary valvuloplasty, prenatal intervention on the atrial septum and fetal cardiac pacing/are feasible during midgestation for improving fetal cardiac function, minimizing fetal loss and enhancing postnatal biventricular repair and neonatal survival. For aortic stenosis with evolving hypoplastic left heart syndrome and pulmonary atresia with intact ventricular septum with evolving hypoplastic right heart syndrome, fetal TI may result in maintenance of a biventricular circulation, thus avoiding single ventricle palliation and its attendant complications. In hypoplastic left heart syndrome with an intact atrial septum, fetal TI may ameliorate in utero pathophysiology and portend a more favorable postnatal prognosis. In all cases, a detailed fetal echocardiographic assessment to identify the appropriate for fetal TI candidate is essential. Future directions of fetal cardiac interventions are a combination of nonsurgical cardiac therapy with minimally invasive surgical techniques, such as robot-guided fetal cardiac intervention, fetoscopic fetal cardiac surgery, and open cardiac surgery.

\section{Fetal Spine: Prenatal Sonographic Views and Detection of the Anomalies}

Danka Miric Tesanic, Eberhard Merz

Polyclinic Gynae Ars,Tomašićeva, Zagreb Centre for Ultrasound and Prenatal Medicine, Frankfurt, Germany

Different 3D sonographic views allow a realistic presentation of the normal fetal spine and also precise detection of the spinal defects. 
Abstracts

Multiplanar view offers a tomographic evaluation of the obtained volume in three two-dimensional perpendicular sectional planes simultaneously presented on the screen. Dorsal and ventral spinal defects can be detected using this mode with great accuracy.

Tomographic ultrasound imaging (TUI), allows the examiner to present the obtained volume data set as multiple images simultaneously displayed on the screen at specific distances. It allows the display of numerous 2D slices from the obtained volume on any of the three orthogonal planes. The opportunity to define the slice thickness (from 0.5 to $10 \mathrm{~mm}$ ) offers the operator the possibility to determine the exact position of the small spinal defect increasing, therefore, prenatal diagnostic accuracy.

Omni view provides a correct and detailed volume analysis of the region of interest. This view can dissect the obtained volume along a sectional plane by using curvilinear or irregular cuts providing in this way a new possibility to study fetal structures, e.g. fetal spine.

Surface view allows demonstration of the fetal surface. Using 3D surface mode open spina bifida, meningocele, as well as ventral spinal defects, could be presented realistically. The additional use of a maximum mode that emphasizes the hyperechoic structures allows the operator visualization and examination of the bony structures and also detection of skeletal anomalies with great accuracy.

Three-dimensional (3D) sonography with its different presentation views has improved the visualization of fetal structures in general, but more so the accuracy of detection of fetal anomalies, in particular, the small defects.

\title{
Three-dimensional Fetal Echocardiography
}

Faris Mujezinović

Perinatology Department, Clinic of Gynecology and perinatology, UKC Maribor, Ljubljanska 5, Maribor, Slovenia

Congenital heart defects are such a group of irregularities, which are often associated with a high degree of perinatal mortality, and in adulthood with a high degree of morbidity.

They represent one of the most common irregularities that can be found in infants, and their spectrum ranges from very mild irregularities, which are often self-cured as, for example, in-utero closure of the small muscular ventricular septal defect (VSD), to very severe, in which prognosis often depends on timely detection of irregularities during pregnancy and on-time delivery of a pregnant woman in the tertiary center, for example in the case of transposition of great vessels.

Detecting as much of as possible cardiac irregularities during intrauterine development, even in the case of consequent intrauterine death, is vital, because in addition to preparing for optimal delivery, we can provide a rationale for the sudden intrauterine fetal death, and enable the assessment of the risk of a recurrence of cardiac irregularities in the following pregnancy.

Despite the importance of timely detection, the degree of the discovery of congenital heart defects in the fetus varies considerably from one country to another. Reasons can be found in an unobtrusive medical examination system from primary to tertiary level, inadequate monitoring of the success of the currently established way of screening, which should give information, how much irregularities are missed, what type, and under what conditions. An additional reason is the number of standard ultrasonic sections, which are used for evaluation if the heart is well developed as well as their adequate documentation. Whether they are done or not, depends also on the level of competence of the gynecologist at the primary level, who are examining the largest part of the pregnant population.

3D ultrasound due to the ability to capture the acquired volume allows the analysis of the heart beyond regular examinations, acquiring more detailed knowledge of the anatomy of the heart and better orientation in its internal structures, and getting too familiar with the most common heart defects.

In recent years, the technology of three-dimensional ultrasound analysis has developed rapidly, but additional knowledge is needed for its use. The lecture presents the basics of 3D heart scanning, which could help not only in routine clinical work but also in increasing the level of detection on the national level.

\section{Advantage of Three-dimensional Sonography in the Assessment of the Corpus Callosum}

\author{
${ }^{1}$ Sonila Pashaj, ${ }^{2}$ Eberhard Merz \\ ${ }^{1,2}$ Centre for Ultrasound and Prenatal Medicine, Frankfurt/Main, Germany
}

The corpus callosum is an essential structure for the normal morphogenesis and function of the fetal brain. Over the last decades, there has been an increasing number of publications describing the benefit of 3D ultrasound in the identification of the corpus callosum. Three-dimensional ultrasound allows acquisition of the volumes which can be stored, reviewed of-line and shared with experts for the second opinion. Furthermore, it permits to reconstruct the planes that are difficult to obtain by 2D imaging, and it allows precise identification of the location and extent of brain anomalies. 


\title{
NORMAL ANATOMY
}

Using the metopic suture or the anterior fontanelle as a window, the normal corpus callosum is correctly demonstrated as a hypoechoic structure with three-dimensional ultrasound when the volume is acquired from a sagittal plane. If the acquisition plane is not a correct median plane, a correction of the three perpendicular planes can be achieved with the three rotation controls. If the volume acquisition starts from an axial plane of the fetal head, the corpus callosum will appear as a hyperechoic structure without clear demarcation from the cavum septi pellucidi. Thus, it is not possible to identify pathological changes of the corpus callosum.

By using the glass-body mode, the pericallosal artery and the callosomarginal artery can be demonstrated exactly.

\section{PATHOLOGIC RESULTS}

The demonstration of the entire corpus callosum (rostrum, genu, body, splenium) and the measurement of the corpus callosum length and the height of the different parts ${ }^{1}$ allow the detection of several corpus callosum anomalies, starting at 18-20 weeks of gestation: agenesis, partial agenesis, hypoplasia, hyperplasia and lipoma of the corpus callosum. ${ }^{2}$

\section{CONCLUSION}

The best way to demonstrate the corpus callosum with 3D ultrasound is when the brain volume is acquired from a sagittal plane, using the anterior fontanelle, metopic suture or anterior part of the sagittal suture as an acoustic window. 3D ultrasound does not permit the detection of corpus callosum anomalies when the volumes are acquired from an axial plane.

\section{REFERENCES}

1. Pashaj S, Merz E, Wellek S. Biometry of the fetal corpus callosum by three-dimensional ultrasound. Ultrasound Obstet Gynecol 2013; 42: 691-698.

2. Pashaj S, Merz E. Detection of Fetal Corpus Callosum Abnormalities by Means of 3D Ultrasound. Ultraschall Med 2016; 37: 185-194.

\section{Congenital Heart Disease, Fetal Growth, and Placental Hemodynamic}

\author{
Damir Roje \\ Head of Perinatal Medicine Unit, Department of Obstetrics \& Gynecology, Clinical Hospital Center Split, University of Split, \\ School of Medicine, Spinciceva, Croatia
}

The yolk sac is the first of the extraembryonic membranes to be vascularized and is responsible for oxygen support to the early embryo. Placenta and its vasculogenesis is developing at the same time but takes complete function at the end of the first trimester.

Timeline of human early placental and heart development overlap and depends on similar regulatory and epigenetic influence. Therefore, it is not surprising that congenital heart diseases (CHD) and placental morphological and/or histological disorders often happen together.

The average fetus with CHD has lower body mass than expected. Intrauterine growth retardation usually starts in the second trimester and is predominantly symmetric.

Biparietal diameter (BPD) and head circumference $(\mathrm{HC})$ are regularly under standard values. Abdominal circumference (AC) is smaller less often, and femur length (FL) almost always follows population standards. Middle cerebral artery (MCA) flow in serial measurements has the most significant changes, while umbilical artery and vein, venous duct, aorta and uterine artery present no clinically important data. The increase of the diastolic component in MCA Doppler recording is interpreted as a compensatory adjustment by local vascular autoregulation. Therefore, the use of relative resistance in the cerebral versus umbilical arterial vasculature as an indication of the brain sparing effect may be inappropriate in the setting of CHD. Complex magnetic resonance imaging techniques showed out that CHD fetus has lower umbilical vein oxygenation, lower oxygen central nervous system supply, and smaller brain size. Different CHD subtypes, and depend on the venous and oxygenated blood mixing rates determinates the importance of the problem.

Recommendations for gynecologists can be separated into two groups. Knowing that fetus have CHD practitioner should perform: (a) detailed US anomaly scan, (b) serial biometrics that includes at least BPD, HC, AC, FL, and fetal weight estimation; (c) US placental examination, (d) should adjust antenatal care protocol in IUGR cases, (e) must be very careful in controversial Doppler finding interpretation. In order to detect antenatal as much CHD as possible: (a) anomaly scan and CHD screening should be part of daily routine, (b) in case of any detected anomalie(s), IUGR, and in all high-risk antenatal screening test with normal fetal chromosome set pregnancies, fetal echocardiography or at least CHD screening by experienced physician should be organized; (c) in case of unexpected Doppler finding and especially in MCA, it is reasonable to consider CHD as a possible cause. 
Abstracts

\section{BIBLIOGRAPHY}

1. Burton GJ, Jauniaux E. Development of the human placenta and fetal heart: Synergic or independent? Frontiers in physiology. 2018 Apr 12;9:373.

2. Camm EJ, Botting KJ, Sferruzzi-Perri AN. Near to one's heart: the intimate relationship between the placenta and fetal heart. Frontiers in physiology. 2018;9:629.

3. Courtney JA, Cnota J, Jones H. The role of abnormal placentation in congenital heart disease; Cause, correlate or consequence. Frontiers in physiology. 2018;9:1045.

4. Kaltman JR, et al. Impact of congenital heart disease on cerebrovascular blood flow dynamics in the fetus. Ultrasound Obstet Gynecol 2005;25:32-6.

5. Linask KK. The Heart-Placenta Axis in the First Month of Pregnancy: Induction and Prevention of Cardiovascular Birth Disease. Journal of Pregnancy 2013;2013:320413.

6. Masoller N, Martínez JM, Gómez O, et al. Evidence of second-trimester changes in head biometry and brain perfusion in fetuses with congenital heart disease. Ultrasound in Obstetrics \& Gynecology. 2014 Aug;44(2):182-187.

7. Peixoto AB, Tonni G, Júnior EA. Changes in biometry and cerebroplacental hemodynamics in fetuses with congenital heart diseases. Journal of thoracic disease. 2016 Oct;8(10):E1282.

8. Ruiz A, Cruz Lemini M, Masoller N, et al. Longitudinal changes in fetal biometry and cerebroplacental hemodynamics in fetuses with congenital heart disease. Ultrasound in Obstetrics \& Gynecology. 2017 Mar 1;49(3):379-386.

9. Rychik J, Goff D, McKay E, et al. Characterization of the placenta in the newborn with congenital heart disease: distinctions based on type of cardiac malformation. Pediatric cardiology. 2018 Aug 1;39(6):1165-1171.

10. Sun L, Macgowan CK, Sled JG, et al. Reduced fetal cerebral oxygen consumption is associated with smaller brain size in fetuses with congenital heart disease. Circulation. 2015 Apr 14;131(15):1313-1323.

\section{Neurophysiologic Development of the Fetus and Fetal Stress}

Aida Salihagić Kadić

Department of Physiology, University of Zagreb Medical School, Zagreb, Croatia

The development of diagnostic strategies to prevent and reduce the burden of perinatal brain damage has become one of the most important tasks of modern perinatal medicine. The development of any such strategy requires the understanding of normal neurodevelopmental processes and their influence on the fetal functional and behavioral patterns, detectable by modern diagnostic methods. Studies carried out using four-dimensional (4D) sonography have shown the fascinating diversity of fetal functions and activities and revealed that fetal movements and behavioral patterns are valuable indicators of the functional and structural brain development. Progression in behavioral complexity begins with spontaneous fetal movements and culminates with presumed preferences for the sound of mother's voice, reflecting maturational events that take place in the brainstem, followed by forebrain structures. Furthermore, the fetus needs a stimulating matrix of movements, sounds, vibrations, and other stimuli as well as a stress-free environment for normal neurodevelopment and the development of other organs and organ systems. The neuroendocrine stress axis operates in the fetus from midgestation and includes the production and secretion of the stress hormones. Fetal glucocorticoid overexposure has not only prenatal and postnatal but also life-long consequences. It has been found that the underlying etiology of some of the most common diseases of the modern society, such as hypertension, obesity, diabetes, cardiovascular diseases, and psychiatric disorders, has been traced in intrauterine environment.

Further, there is experimental evidence that increased maternal care and environmental enrichment can compensate for prenatal stress-induced effects. Recent data have shown gender differences in vulnerability to fetal stress and a higher risk of depressive symptoms, schizophrenia, autism, and ADHD in boys than in girls. Finally, further investigations supported by the new imaging techniques should open a new perspective for the development of prenatal diagnostic strategies to detect and/or prevent severe neurologic disorders as well as minimal cerebral dysfunctions.

\section{Three-vessel Trachea View is Good Enough at Routine Fetal Heart Examination?}

Cinat Sen

Perinatal Medicine Center, Memorial Hospital, Istanbul, Turkey

There are three steps for fetal heart examination: Abdominal situs, four-chamber view, three-vessel trachea (3VT) view. Detection rates can be optimized by performing a complete examination of the fetal heart, recognizing that the four-chamber view is much more than a simple count of cardiac chambers. Additionally, the 3VT-view is, therefore, an important step to improve detection of CHD up to 85. The evaluation of 3VT view increases the detection rates for major heart malformations 
above those by the four-chamber view alone such as tetralogy of Fallot, transposition of the great arteries, double outlet right ventricle and truncus arteriosus.

Normal 4-chamber view and outflow tracts together with V-shape 3-vessel view can rule out most and major fetal heart defect at the time of routine ultrasound examination. A clear picture of (a) transvers abdominal section with stomach and aorta on the left and inferior vena cava on the right-anterior, (b) four chamber, (c) aortic route arising from left ventricle continuing with septum and aortic wall, (d) main pulmonary artery arising from right ventricle with branching which is crossing aorta, V-shape 3VT view should be obtained and stored in printed and/or electronic format. If anyone can show those pictures like a normal appearance, no one can blame because he or she has done the examination in a structured way as a good practice. If any of those sections is not able to show the clear-normal appearance of these structures which can be abnormal or suspected, this is the first step of the diagnostic work-up. Those cases are subjected to fetal echocardiography as perinatal heart examination. Then definitive diagnostic examination is going to be done. Routine fetal heart examination is being done to explore the normality with a normal four-chamber view and great vessels. If it is not clear, then, next step should be fetal echocardiography as perinatal heart examination to rule out any fetal heart abnormality and for differential diagnosis.

Fetal heart examination can and should be done according to the condition explained above and can be performed from 12 weeks onward. The appearance of the abnormality can be early or late pregnancy according to the type and blood circulation in the fetal heart abnormality in first, second and even third trimester of pregnancy. More detailed information about the diagnosis of certain heart disease can be found in the other related chapters.

\section{Monochorionic Twin Pregnancies: Laser Surgery}

Cihat Sen

Perinatal Medicine Center, Memorial Hospital, Istanbul, Turkey

Twin-to-twin transfusion syndrome and selective-IUGR are the most common clinical problems. Monochorionic twins share the same placenta and frequently have anastomotic vessels between two fetal circulations. Time to time a little amount of blood exchange is possible but in a balance. In some cases, about $10-15 \%$ of monochorionic are showing twin-to-twin transfusion syndrome. In TTTS situation, exchange of blood between two fetuses can become unbalanced, and one fetus may supply of some blood to recipients (the same cotyledon may be supplied by the donors and vein goes to other (recipient). Mostly TTTS cases are apparent clinically at 16-26 weeks of gestation.

\section{TWIN OLIGOHYDRAMNIOS-POLYHYDRAMNIOS SEQUENCE}

Twin oligohydramnios-polyhydramnios sequence (TOPS) is one type of twin-twin transfusion syndrome and seen about $10 \%$ of and accounts for the majority of morbidity and mortality monochorionic pregnancies. Fetoscopic laser photocoagulation has been shown the optimum management option over amnioreduction in a randomized controlled trial. Laser surgery is offered for Quintero stage II-IV and stage I for symptomatic polyhydramnios, recipient cardiomyopathy based on echocardiographic examination, or shortened cervix. Fetoscopic surgery is performed with local anesthesia or regional anesthesia, in some centers with intravenous sedation. Special attention is given to procedure complications such as preterm premature membrane rupture ( $30 \%$ cases), intraabdominal leakage of amniotic fluid ( $3 \%$ ) and placental abruption $(1 \%)$.

\section{TWIN ANEMIA-POLYCYTHEMIA SEQUENCE}

Twin anemia-polycythemia sequence (TAPS) is another type of twin-twin transfusion syndrome which is caused by chronic and unbalanced blood transfusion via tiny anastomotic vessels in monochorionic pregnancies. It is characterized by polycythemia in the recipient and anemia in the donor twin with normal amniotic fluid volume. This phenomenon in the fetus is diagnosed by peak systolic velocity in middle cerebral artery greater than 1.5 MOM in donor twin and less than $1.0 \mathrm{MOM}$ in recipient twin. Twin anemia-polycythemia has five stages according to the MOM value of MCA peak systolic velocity, Doppler abnormality, hydrops, and fetal demise as in TOPS staging.

\section{SELECTIVE INTRAUTERINE GROWTH RETARDATION}

Selective intrauterine growth retardation (sIUGR) is one of the major complications in monochorionic pregnancies which has increased the risk of fetal death and neurological damage. The incidence of sIUGR is approximately $10-15 \%$ of monochorionic pregnancies. It is diagnosed by abdominal circumference less than 2SD according to gestational age, or some investigators use an estimated fetal weight below the 10th percentile (with or without weight discordance-greater than $25 \%$ ).

\section{MONOAMNIOTIC TWIN PREGNANCIES}

Monoamniotic twin pregnancies are rare cases and reported to occur approximately in 1\% of monochorionic twin pregnancies. Monoamniotic pregnancies are at high risk for perinatal mortality about $10-40 \%$ of cases and with a prevalence of $26 \%$ congenital 
Abstracts

abnormality. Most of the mortality is due to preterm delivery and congenital anomalies, not due to cord entanglement because the cord entanglement was accepted for a long time as the main cause of fetal mortality.

To bear in mind, the placenta in monoamniotic pregnancies have significant numbers of anastomoses (deep and superficial) comparing to uncomplicated monochorionic diamniotic pregnancies. If a sign of cord entanglement is detected, fetoscopic laser cord coagulation and cord transection are recommended for reducing a cord accident.

\title{
TWIN REVERSED ARTERIAL PERFUSION
}

In twin reversed arterial perfusion (TRAP), the living fetus is supplying blood to a nonviable twin pair "acardiac twin" through an artery-artery anastomosis which the acardiac twin has blood in a reversed way from this single artery from the living fetus. This is a kind of shunt circulation and causes a high output heart failure in the living fetus (the pump twin). For this reason, twin reversed arterial perfusion cases can be treated with intravital laser surgery under ultrasound guidance or fetoscopic fetal laser surgery to coagulate the anastomotic vessels. It is better to make intervention early around 16 weeks of gestation.

\section{Diagnosing Fetal Syndromes by 3D/4D Ultrasound: Are there Any Improvements?}

Lara Spalldi Barišić

Ian Donald School of Medical Ultrasound, Croatian branch, "Poliklinika Veritas" and DZZI Zagreb, Zagreb, Croatia

\section{FROM PIECE TO A COMPLETE PUZZLE}

A dysmorphic feature is a difference in body structure. It can be an isolated finding, part of the normal human variation in an otherwise normal individual, or it can be related to a congenital disorder, birth defect or genetic syndrome. Dysmorphic features can vary from isolated, mild anomalies and minor cosmetic imperfections (such as polydactyly) to severe congenital anomalies (such as holoprosencephaly). In some cases, dysmorphic features are part of a larger clinical picture, sometimes known as a sequence, association or syndrome.

A syndrome is a pattern of multiple anomalies thought to be pathologically related, the particular combination of major (essential) and minor (may be absent) criteria.

So, why searching for fetal syndromes?

Early diagnosis is very important especially in the delineation of the best care for the patient, prognosis, likelihood of other abnormalities, identifying correct recurrence risk and the best approach to monitor future pregnancies. Being able to provide as clear information as possible is of great importance to avoid confusion in parents as well as healthcare providers, to make a management plan and to put everything in perspective.

Recognizing the patterns of fetal malformations is extremely useful for sonologist, practitioners providing a prenatal diagnosis. Ultrasound findings of abnormalities and patterns of more common fetal syndromes, as well as some less common fetal syndromes, are lined up in this presentation.

Technological advances in ultrasonography, particularly the introduction of high definition 3D and 4D ultrasound allowed us to study fetal anatomy in great detail in very early stages of fetal life, which on their hand helped us to detect fetal abnormalities easier and even earlier than ever before.

Beside fetal anatomy, we are now even able to study a function of some systems and fetal behavior. Fetal behavioral patterns are directly reflecting the development and the maturational process of fetal CNS. KANET test is the first method that attempted to use $4 \mathrm{D}$ US to asses and combine different parameters of fetal behavior and form a scoring system to determine their neurological status.

Currently, we are being able to detect not only structural abnormalities but also fetal functional and behavioral abnormality patterns (by 3D and 4D ultrasound technology) related to a fetal syndrome in the era of prenatal diagnosis.

To understand and to resolve many questions we do require a multidisciplinary approach. At Ian Donald School of Medical Ultrasound, our teams of doctors all over the world are powered and guided by the same goal: to continuously embrace different innovation in cutting-edge technology, to learn and develop new skills, share the knowledge and to implement it in a way that can improve the lives of once that need it the most.

\section{Surgically Correctable Congenital Malformations: Role of Neonatologist}

\author{
Milan Stanojevic \\ Department of Obstetrics and Gynecology Medical School University of Zagreb \\ Neonatal Unit, University Hospital "Sveti Duh" Zagreb, Croatia
}

Congenital malformations are a significant cause of neonatal and infant mortality in developed and in developing countries. In Croatia, the congenital malformations were the cause of $5.4-16.5 \%$ of fetal and $20.2-33.3 \%$ of early neonatal deaths. Congenital 
malformations constitute $31.1 \%$ of infant mortality in Croatia during the five-year period, being in the second place behind the causes arising in the perinatal period. Finding that the fetus has a severe congenital malformation could be very stressful for the couple because they are expecting a perfect baby. Parents should be counseled objectively taking into account parental cultural, religious and social differences in the informed decision-making process, without the influence of the counselor's attitude (nondirective counseling). For many severe congenital malformations data on the long-term outcome are missing, which may further frustrate parents and healthcare professionals from which parents expect comprehensive and appropriate information.

The aim of this paper is to define the long-term outcome based on the example of some severe congenital malformations taking into account ethical, social, economic and other aspects that can occur in patients with severe congenital malformations. In particular the concept of "futility of treatment" will be discussed with some examples of so-called lethal congenital malformations. Parental counseling modalities will be discussed based on the data from the literature on prenatal diagnosis, treatment, and long-term outcome for congenital diaphragmatic hernia, open spina bifida, hypoplastic left heart syndrome, omphalocele, and gastroschisis. Despite the progress of perinatal medicine, neonatal and surgical care, quality research on the long-term outcome of children with major congenital defects are still lacking. This kind of research would allow better prenatal counseling which always should include ethical aspects allowing parents the adoption of the evidence-based informed decision.

\title{
Role of Ultrasound in the Diagnosis of the Most Frequent Fetal Anomalies in the First and Second Trimesters of Pregnancy
}

Selami Sylejmani

\author{
Medical School University "Fehmi Agani", Đakovica, Kosovo
}

\section{INTRODUCTION}

According to the World Health Organization (WHO), the abnormality means any anatomical anomalies of development, visible during clinical examination or autopsy (if the baby is dead), but it is present at birth or is observed during the stay of the child in the delivery room. Fetal congenital abnormalities occur in approximately 3-5\% of infants. Organ malformations occur during organogenesis, respectively from the 2 nd to the 12 th week of pregnancy. Causes can be genetic, infectious, environmental or foodrelated. In most cases, it is difficult to ascertain the exact cause of anomalies. Inborn abnormalities can be defined as structural or functional, which appear during intrauterine life. These can be diagnosed before birth, at birth, or may exhibit symptoms later in life. Birth abnormalities are a major cause of newborn deaths, chronic illnesses, or childhood disability in the world. The most severe congenital abnormalities are those of the heart, followed by the abnormalities of the neutral tube and the urinary tract. Perinatal diagnostics include a range of diagnostic methods with the help of which we highlight or exclude the chromosomal aberrations at the fetus, a significant number of congenital metabolic disorders and hereditary diseases associated with the $X$ chromosome, and a range of fetal morphological anomalies. Sonodiagnostics has an important place in diagnosing these anomalies. Routine ultrasound examination is an integral part of prenatal care if resources are available and if there is possible access. It is usually done in the second trimester, although the routine ultrasound is increasingly available in the first trimester, particularly in circumstances where there are abundant resources. Constant technological achievements, including the transvaginal ultrasound with high frequency, have enabled the resolution of ultrasound imaging in the first trimester, to evolve to a level at which the early fetal development can be assessed and monitored in details.

\section{PURPOSE OF THE PAPER}

The purpose of the paper is to highlight the role of sono diagnostics in diagnosing the prenatal most common fetal anomalies based on our experience.

\section{GENERAL INFORMATION}

Since the ultrasound is mechanical energy, without accumulative effect, there is no work-up to date to show that its emission has shown a harmful effect in the development of the fetus. These advantages have enabled us to monitor the pregnancy in the earliest stages of its development. This advantage has been crucial for the sono diagnostics to be accepted as a prenatal diagnostic method, which was spread very quickly, with the sole purpose of helping reduce the perinatal mortality and morbidity. Although ultrasound is in clinical use for over 45 years, the greatest progress has been made in the past 15 years, with the introduction of high-frequency probes, that dramatically improved sensitivity and resolution, Color Doppler used to assess maternal and fetal circulation, and 3D and 4D ultrasound dimensions and lastly the live HD ultrasound. These advantages, together with the implementation of digital technology, and the development of cheap portable scanners, have resulted in a huge increase in the application of ultrasound in general and obstetrics in particular. The new sonodiagnostics conventional methods such as the high-resolution ultrasound, 3D and 4D ultrasound and HDlive ultrasound, have enabled the advancement of this diagnostic method. The transvaginal ultrasound with high resolution has opened new opportunities for a new scientific discipline called sonoembryology, which deals with the study of embryonic development. The sonoembryology has shown its value by early detecting certain congenital anomalies of the fetus. This method of ultrasound diagnostics has taught us at least two things: firstly, that in the 11th week, it will come to the turn of bowel in the peritoneal area, and secondly, that the greatest measure of bowel 
Abstracts

outside the abdominal wall happens in the 10th week (a physiological hernia). Therefore, with the new generation of ultrasound equipment, there can be diagnosed numerous anomalies starting from the 10th week of pregnancy. The color Doppler is also a technical breakthrough which further helps in the diagnosis of congenital anomalies. The Pulse-Doppler enables a timely detection of fetal chronic hypoxia. There have been more than 20 years since the three-dimensional (3D) ultrasound was first applied in clinical practice, and now it comes to $4 \mathrm{D}$ ultrasonography coupled with impressive images of live HD technology. The 4D ultrasound, is a complement to 3D ultrasound, adding the real-time component. With this diagnostic method, we can follow the fetal movements, and better analyze abnormalities of many systems.

\title{
CONCLUSION
}

The conventional sonodiagnostics, aided by additional technologies such as color Doppler, 3D/4D ultrasound, and lastly HDlive ultrasound, is a powerful tool in diagnosing the prenatal most common fetal anomalies, and distorted and pathological pregnancies. Without the help of sonodiagnostics, the timely diagnosis of congenital fetal anomalies is impossible.

\section{Possibilities of the Fetal Echocardiography from the Pediatric Cardiologist's Viewpoint}

Dalibor Šarić

Congenital heart defects (CHDs) are the most common congenital malformations with a prevalence of 8 per 1000 live births. They are the leading cause of perinatal and infant deaths caused by congenital malformation. Prenatal detection of CHD is crucial to improving the outcome in critical CHD, especially when systemic or pulmonary circulation depends on the patency of the arterial ductus. Antenatal screening for fetal cardiac abnormalities was introduced over 25 years ago. Over the past two decades, the possibility of examination of the fetal heart and cardiovascular system has evolved considerably, mostly as a result of advances in imaging technology. Advanced technology has allowed more accurate and early detection of cardiac abnormalities and has also improved the care and outcome of fetuses with severe CHDs or arrhythmias.

Echocardiography is the best method currently available for the diagnosis of structural heart defect, arrhythmias, and hemodynamic abnormalities in the fetus.

It must be remembered that fetal echocardiography requires the use of high-quality ultrasound machines and, in particular, an examiner who can perform the comprehensive cardiac examination. High risk for fetal heart defects and the suspicion of a cardiac abnormality on obstetric ultrasound are indications for referral to pediatric cardiology center for a detailed fetal echocardiogram.

In experienced hands, fetal echocardiography can detect most congenital heart lesions at 18-20 weeks of gestation. Anatomical details of most CHD in fetuses can be provided by two-dimensional (2D) ultrasound. Notable exceptions are the atrial septal defect, because of the extreme thinness and the presence of the patent foramen ovale of the atrial septum in the fetus, and the arterial duct, which is a normal finding. Some defects can also evolve or progress during fetal life, making a definitive diagnosis early in gestation difficult.

Echocardiographic assessment of fetal arrhythmias is usually accomplished using M-mode and Doppler techniques. Echocardiography not only establishes the kind of arrhythmia present but can also identify associated structural and functional abnormalities of the fetal heart as well.

Prenatal diagnosis allows for planned management of the fetal heart disease prenatally and postnatally. It gives us the opportunity to discuss the findings with parents and describe them the management plan. Prenatal detection of CHD provides a chance for delivery at a tertiary care center where the pediatric cardiologists and cardiac surgeons are available. It can also identify patients for in utero cardiac interventions.

\section{First Trimester: Early Ultrasound Examination}

\author{
Suada Tinjić \\ Gynecological Clinic, Korak do Života \\ Director of lan Donald School of Tuzla, Bosnia and Herzegovina
}

\begin{abstract}
The intensive development of ultrasonic diagnostics, with a special emphasis on the detailed evaluation of the first trimester using transvaginal sonography, enabled the shift of ultrasonic screening of chromosomal aberrations from the second to the first trimester. Vaginal high-frequency probes allow high resolution of anatomic details during early embryonic development.

The first trimester, mostly defined as the first 100 days of pregnancy, is marked by many important sights announcing the ultimate outcome of pregnancy. Although a positive pregnancy test is most likely to be intrauterine, the production of human chorionic gonadotrophin (hCG) also occurs in tumors (dysgerminomas, choriocarcinoma), or in insufficiently developed pregnancies, such as ectopic pregnancy, damaged fallopian tube or molar hydatidosis. Other early complications and pregnancy failures, such as retrodecidual hematomas, missed abortion, incomplete abortion, retained conception products, are likely to be accompanied by
\end{abstract}


clinical symptoms such as lower abdominal pain and vaginal bleeding. Early embryo and early fetus development, its anatomy and first movement patterns were explored using 3D and 4D ultrasound. Another area of remarkable expansion was the study of the first trimester between 11 and 13/6 weeks of pregnancy. This includes not only early diagnosis of fetal structural anomalies, and screening of aneuploid fetuses such as trisomy 21,18 and 13, but also offers a likelihood of hypertensive preeclampsia and intrauterine growth (IUGR).

Ultrasound examination in the first trimester should be done by an accredited physician with good knowledge of embryology, development, and changes during pregnancy and good knowledge of distinguishing between normal and abnormal changes.

Before the ultrasound examination, we need information on the patient, life expectancy, parity, conception method, history of gynecological and other diseases as well as family history of chronic, acquired and genetic diseases. Then the date of the last menstrual cycle due to amenorrhea length correlation and ultrasound estimation of expected delivery term.

Transvaginal ultrasound in early pregnancy, we confirm the diagnosis of pregnancy, we distinguish between normal pregnancy and outbreaks of pregnancy, confirm the vitality of fetal and cardiac action, basic biometrics and gestational age, differentiation of single and multiple pregnancy, early pregnancy complications and possibility of miscarriage, ultrasound diagnosis marker for chromosomal aberrations and malformations. We investigate uterine malformations (septum, miomi), changes in the cervix (cervicometry), review of adnexa and diagnosis of pathological changes in adnexas, as well as early pregnancy transvaginal color Doppler monitoring (uteroplacental flow, intravenous flow, blood through the umbilicus as well as the embryonic and early fetal blood flow, the flow through the yolk sack and the duodenum ductus). Transvaginal high-frequency probes (9-12 MHz) yield good results on the structure and anatomy of the small pelvis.

\title{
BIBLIOGRAPHY
}

1. Asim Kurjak et al. Ultrasound in Gynecology and Perinatology. 2007;328-343.

2. Kurjak A, Chervenak FA, editors. Donald School Textbook of Ultrasound in Obstetrics \& Gynaecology. JP Medical Ltd; 2017 Jul 17. p. 106-107.

\section{Neurodevelopmental Outcome of up to Three-years-old Children Born from High-risk Pregnancies: A Prospective Study}

\author{
${ }^{1}$ Sanja Tomasović, ${ }^{2}$ Milan Stanojević, ${ }^{3}$ Leila Mitrović, ${ }^{4}$ Damir Žalac \\ ${ }^{1}$ Department of Neurology, University Hospital “Sveti Duh”, Zagreb, Medical School, “Josip Juraj Strossmayer” \\ University, Osijek, Croatia \\ ${ }^{2}$ Department of Obstetrics and Gynecology, Neonatal Unit, University Hospital "Sveti Duh" Zagreb, Croatia \\ ${ }^{3}$ Korcula Outpatient Health Center, Korcula, Croatia \\ ${ }^{4}$ Private Hospital for Obstetrics and Gynecology "Podobnik", Zagreb, Croatia
}

Prenatal fetal neurological damage can be identified by the introduction of four-dimensional ultrasound (4D US) by application of new prenatal neurological screening test-Kurjak antenatal neurodevelopmental test (KANET). Abnormal KANET test in high-risk fetuses with postnatal biochemical and clinical signs of hypoxia may make predictable development of postnatal mild to severe psychomotor retardation including cerebral palsy (CP). Application of new prenatal test could probably contribute to the prenatal prediction of postnatal neurodevelopmental impairment. ${ }^{1-4}$

The aim of our research was to verify the new method of functional assessment of the central nervous system (CNS) for prenatal detection of brain impairment. The aim could be achieved by objective scoring during application of prenatal KANET test in fetuses from high-risk pregnancies, and postnatal follow up of infants and children up to three years of age. ${ }^{2-6}$

From January 1 to December 31, 2009, 50 infants from high-risk pregnancies delivered at the Department of Obstetrics and Gynecology, University Hospital "Sveti Duh" were included to the prospective study. Inclusion criteria were as follows: previous child with CP; mother's conditions: diabetes mellitus types 1 and 2, thyroid gland impairment, arterial hypertension diagnosed before pregnancy, drug and/or substance abuse, thrombophilia, sideropenic anemia, epilepsy, gestational diabetes, Rh incompatibility, threatened preterm labor, preeclampsia, intrauterine infections, TORCH infections, cholestasis in pregnancy; fetal conditions: structural and numerical chromosomal abnormalities, polyhydramnios, intrauterine growth restriction, abnormal cardiotocography and/or Doppler findings, and fetal asphyxia in labor. In all fetuses KANET test has been applied at least two times during the second and the third trimester of pregnancy. In fetuses with abnormal KANET scores, the postnatal neurological assessment was performed using Amiel Tison neurological assessment at term (ATNAT), assessment of general movements (GMs) at writhing and fidgety age, and assessment of developmental milestones according to uturi .,8 The children were followed until the age of 3 years.

The results showed that birth weight and gestational age of high-risk infants and infants from the general population were significantly different, while Apgar scores after 1 minute and 5 minutes were not. Fewer infants were born by cesarean section from the investigation group than from the general population, which could be caused by small sample size and difference in gestational age between the groups. Median of KANET score in the investigated group was 15 with the range from 13 to 19 , while the mean was 15.1 and standard deviation of 1.1. KANET scores were not influenced by the birth weight, gestational age, and sex of the fetus. Correlation between KANET scores and mode of delivery, gestational age, sex, Apgar score in the 1st and the 5th minute was not statistically significant. There was no correlation between KANET, ATNAT, and GMs. Statistically significant 
difference was found between Apgar scores in the 1st and 5th minute by t-test $(t=-2,281 ; p=0,027)$. It was found that the 1 st minute Apgar scores differed significantly between male and female infants due to different gestational age $(t$-test $p<0.001)$. Similar results were obtained for 5 minutes Apgar score. The correlation between KANET score and: sex, mode of delivery, gestational age, birth weight, Apgar score after 1 and 5 minutes, ATNAT, GMs and postnatal assessment of development at 3, 6, 9, $12,18,24$ and 36 months, was not statistically significant.

When testing influence of KANET and the 1st minute Apgar score on developmental milestones at different ages, it was shown that KANET was significantly influencing the postnatal assessment of development at 3 and 24 months, while individual influence of the 1st minute Apgar score and combined influence of KANET and 1st minute Apgar score was not statistically significant in any observed age.

KANET was significantly influencing the postnatal assessment of development at 3 and 6 months, while the individual influence of the 5th minute Apgar score and combined influence of KANET and the 5th minute Apgar score was not statistically significant in any observed age.

There was no statistical influence of KANET and ATNAT individually and when they were combined on the assessment of development at the age of 36 months.

Due to small sample size (which was the limitation of this study), this investigation did not prove the existence of any connections between KANET and other observed variables. The correlation between KANET and other observed variables in infants and children was not found either for the same reason. It was possible to detect impaired (not always pathological) neurodevelopment in fetuses by application of KANET, however in only one fetus from our investigated group the score was 13 which is considered mildly abnormal compared to normal scores of 14 and above. In this investigation KANET test applied in high-risk pregnancies was not proven enough sensitive and specific, which could be caused by a relatively small sample size and imprecise definition of prenatal neurological risk. Expectedly, a statistically significant and positive correlation between ATNAT and GMs was found in postnatal assessment. The results of this investigation suggest that neither of the investigated variables was predictive for the future neurodevelopmental outcome of infants born from high-risk pregnancies. Up to now, KANET was proved to be very reliable in the assessment of definitely abnormal fetuses, while its potential in fetuses with mild impairment was questionable in this study due to small sample size.

\section{REFERENCES}

1. Kurjak A, Predojevic M, Stanojevic M, et al. The use of $4 \mathrm{D}$ imaging in the behavioral assessment of high-risk fetuses. Imaging in Medicine 2011;3:557-569.

2. Stanojevic M, Kurjak A, Salihagic-Kadic A, et al. Neurobehavioral continuity from fetus to neonate. J Perinat Med 2011;39:171-177.

3. Tomasovic S, Predojevic M. 4D ultrasound-medical devices for recent advances on the etiology of cerebral palsy. Acta Inform Med 2011;19:228-234.

4. Miskovic B, Predojevic M, Stanojevic M, et al. KANET test: Experience of Zagreb Group. Donald School J Ultrasound Obstet Gynecol. 2012;6:166-170.

5. Stanojevic M, Zaputovic S, Pavicic Bosnjak A. Continuity between fetal and neonatal neurobehavior. Semin Fetal Neonat Med 2012;17:324-329.

6. Kurjak A, Talic A, Honemeyer U, et al. Comparison between antenatal neurodevelopmental test and fetal Doppler in the assessment of fetal well being. J Perinat Med 2013;41:107-114.

7. Amiel-Tison C. Update of the Amiel-Tison neurological assessment for the term neonate or at 40 weeks corrected age. Pediatr Neurol 2002;27:196-212.

8. Cuturic N. Istrazivanja provedena u nas. In: Cuturic N. Psihomotoricki razvoj djeteta u prve dvije godine zivota. Jastrebarsko: Naklada Slap; 2007:19-23. 


\title{
Prenatal Diagnosis and Outcomes of Complex Congenital Heart Disease in Kosovo
}

\author{
${ }^{1}$ Ramush Bejiqi, ${ }^{1}$ Ragip Retkoceri, ${ }^{1}$ Rinor Bejiqi, ${ }^{1}$ Aferdita Mustafa, ${ }^{1}$ Arlinda Maloku, ${ }^{2}$ Hana Bejiqi \\ ${ }^{1}$ Pediatric Clinic, University Clinical Centre of Kosovo, Prishtina, Republic of Kosovo \\ ${ }^{2}$ Main Centre of Family Medicina, Prishtina, Republic of Kosovo
}

\section{INTRODUCTION}

With a prevalence of 6-13 per 1000 birth lives congenital heart anomalies (CHA) are the most common congenital disorders in newborn and leading factor in neonatal death. Fetal echocardiography (FE) is broadly defined as a detailed sonographic evaluation that is used to identify and characterize fetal heart anomalies (FHA) before delivery. Improving FE in Kosovo as the youngest country in Europe with still high natality and mortality rate in early detection of heart anomalies contributes to planning delivery and in reducing mortality as a consequence of HA.

The aim of the presentation is to present the impact of prenatal diagnosis and postnatal treating fetuses and children with complex CHA in Kosovo as a country with limited human and technical resources.

\section{METHODS}

Retrospectively, we analyzed medical reports and data, obtained from the FE,

\section{RESULTS}

During this period January 2015 and December 2017 FE in 1413 pregnancies were performed (mean 1.5); the age of gestation was from 14 to 32 weeks (mean 23 weeks). Of them, 264 were pregnant with twins (12.5\%), and 13 pregnancies $(0.6 \%)$ were with three fetuses (all from IVF). Indication for FE in all multiple pregnancies and 514 other was actual obstetric findings, in 218 indications were positive history for $\mathrm{CHA}$, in 123 indications was an infection and use of medication during the pregnancy and, in other 399 pregnancies indication was the age of parents, diabetes or self-initiative of parents to do FE. In 243 pregnancies (11.55) CHA was registered, where 68 had been complex. Twenty-two of them were with the complete atrioventricular canal, 17 were with hypoplastic left heart syndrome, in 12 were tricuspid atresia, other 17 were with tetralogy of fallot or double outlet right ventricle. From 68 pregnancies with CHA, despite advice to continue the pregnancy only 9 continued pregnancies (all were with multiple fetuses where another one was healthy).

\section{CONCLUSION}

Despite application, the FE and accurate diagnosis most pregnant women interrupted pregnancy as a consequence of CHA. Situation exacerbates in the fact of lack of pediatric cardiac surgery services on Kosovo. 